\title{
Dynamics of Granular Systems
}

\author{
Hans J. Herrmann ${ }^{(1,2)}$, S. Luding ${ }^{(1)}$, C. Cafiero ${ }^{(2)}$ \\ (1) ICA-1, University of Stuttgart, Pfaffenwaldring 27, 70569 Stuttgart, Germany \\ (2) PMMH, ESPCI, 75231 Paris, France
}

\begin{abstract}
Granular systems show a pronounced deviation from classical Boltzmann statistics of several aspects. In particular, we review some findings on anomalous velocity distributions.
\end{abstract}

Key words: granular materials, velocity distribution

\section{Introduction}

Granular media are dissipative systems. The collisions between the grains are inelastic and therefore energy is intrinsically lost within the system while it is in motion. In order to explore some of the fundamental resulting effects on the statistical properties of such systems, let us idealize a granular medium by a set of $N$ spherical particles, all being identical and having only the exclusive volume hard-core potential as interaction. In addition, one can imagine the particles to rotate and to be able to transfer angular momentum from one to the other during the collision. In that case, it is important to consider also the tangential frictional properties and the tangential dissipation. The inelastic properties are well described by the restitution coefficient material constants. For the time being let us ignore gravity.

Physical realization of such idealized systems are for instance in some sense the interstellar dust [1] and in two dimensions experiments on air tables. But also experiments [2-7] under less idealized conditions can be performed and have given insight about the statistics of such dissipative gases. It has been shown by Goldhirsch and Zanetti [8] that such a dissipative gas undergoes an instability of clustering. The loss of homogeneity of the system has been made responsible for anomalous velocity distributions [9]. It is the issue of this paper to review some of the origins of deviations from Maxwell distributions, looking in particular at the steady state in the case of the homogeneously driven system. 


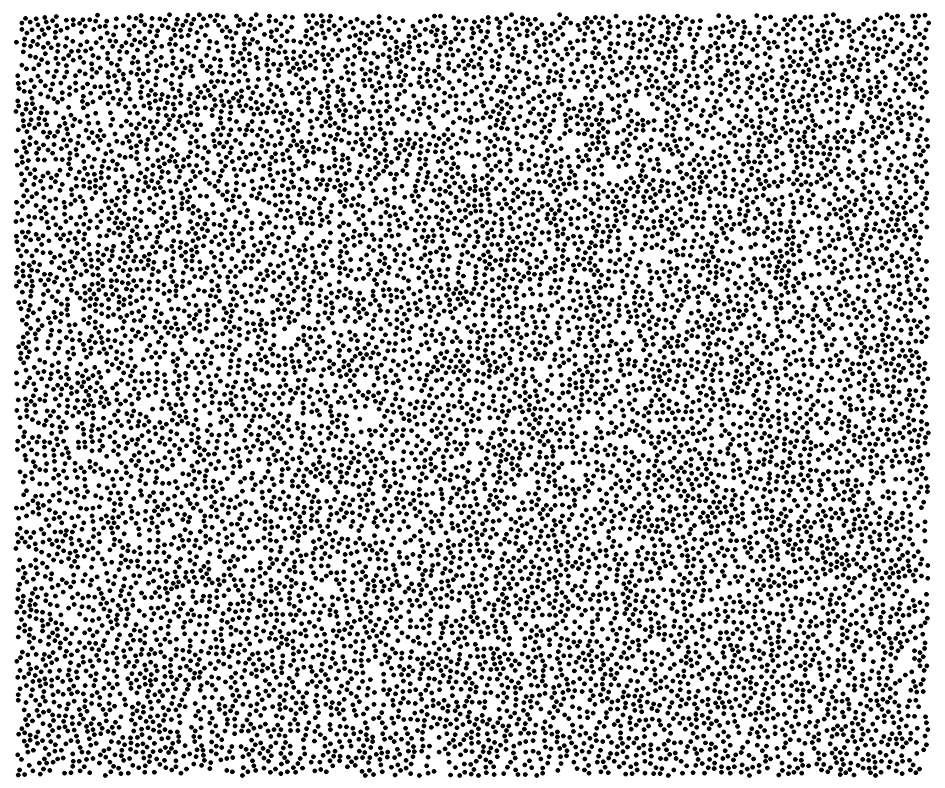

Fig. 1. Initial configuration: 11000 particles in a box with periodic boundary conditions.

Equally puzzling non-Boltzmann distributions are also found in dense granular systems. These have been experimentally idealized in the two-dimensional experiment by Behringer and collaborators $[10,11]$. These systems of sheared disks have also been simulated numerically confirming the experimental findings $[10,12]$. We will also review the findings of these measurements concerning the velocity distributions.

\section{Velocity distributions in the dissipative gas}

Let us consider, as described above, a simple system of $N$ equal spheres in a box with periodic boundary conditions. In Fig. 1 we see such a system of $N=11000$ particles. When two particles collide, the normal component of the relative velocity is reduced by a factor $r$, the normal restitution coefficient which lies between 0 and 1. $r=1$ is the elastic case one encounters in an atomic or molecular gas. The tangential component of the relative velocity between the two particles is changed by a factor $r_{t}$, the tangential restitution coefficient. This tangential restitution coefficient contains two physical effects, namely dissipation and Coulomb friction. Typically each of both effects is described by its own value and $r_{t}$ is chosen to be the minimum of the two. Rotating particles have as additional degree of freedom the angular velocities. The relative tangential velocities between two grains at the contact point is then the sum of the translational and rotational components. 


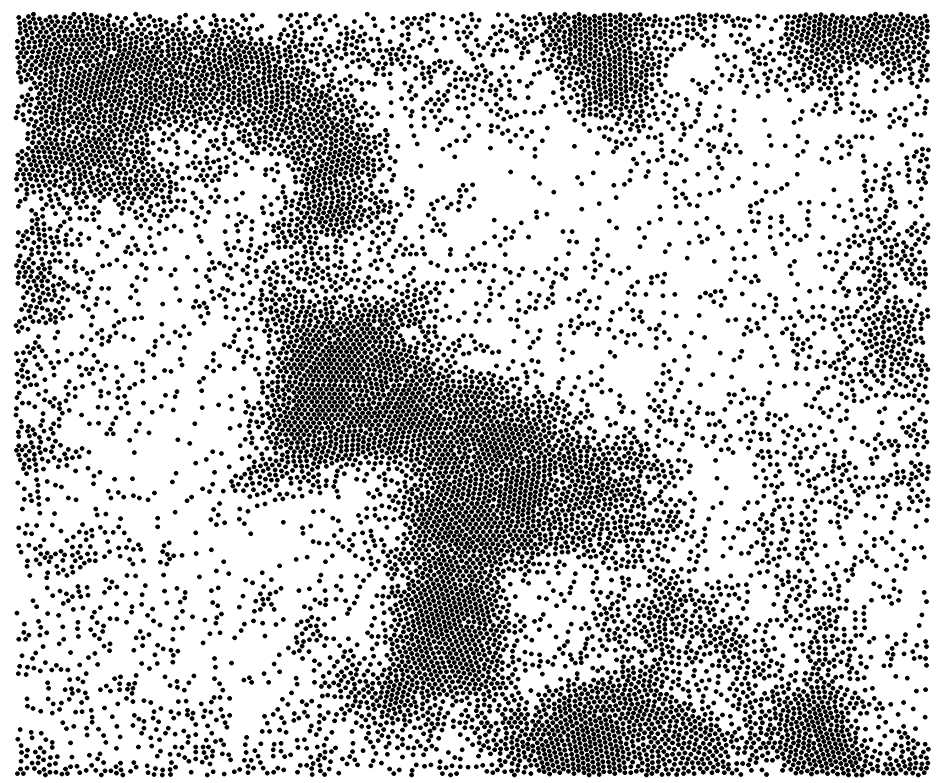

Fig. 2. Steady state for $\delta=1, r=0.97$, see eq. (4).

There exist a variety of possibilities to simulate such systems, the most efficient one being an event driven algorithm using look-up tables. Simulations of such systems have been performed by many authors $[6,8,13-15]$.

If such a system is initialised with a certain distribution of velocities for the particles and no further energy is put into the system, then it cools down and the energy decreases like

$$
E \propto \frac{1}{t^{2}}
$$

in time in agreement with the kinetic gas theory [16]. After some time one observes the appearance of clusters due to the instability mentioned above, similar to those seen in Fig. 2. Denser regions have more collisions and therefore more dissipation. Consequently the pressure decreases and a flux appears from outside into the already dense region. In this clustering regime [15] the energy decreases in time only like

$$
E \propto \frac{1}{t}
$$

The velocity distribution also changes. Inside the clusters the particles are particularly slow and between the clusters the mean free path is very long and particles of comparatively large velocities can exist. Therefore, as seen in the schematic diagram of Fig. 3, the distribution of velocities in the cluster- 


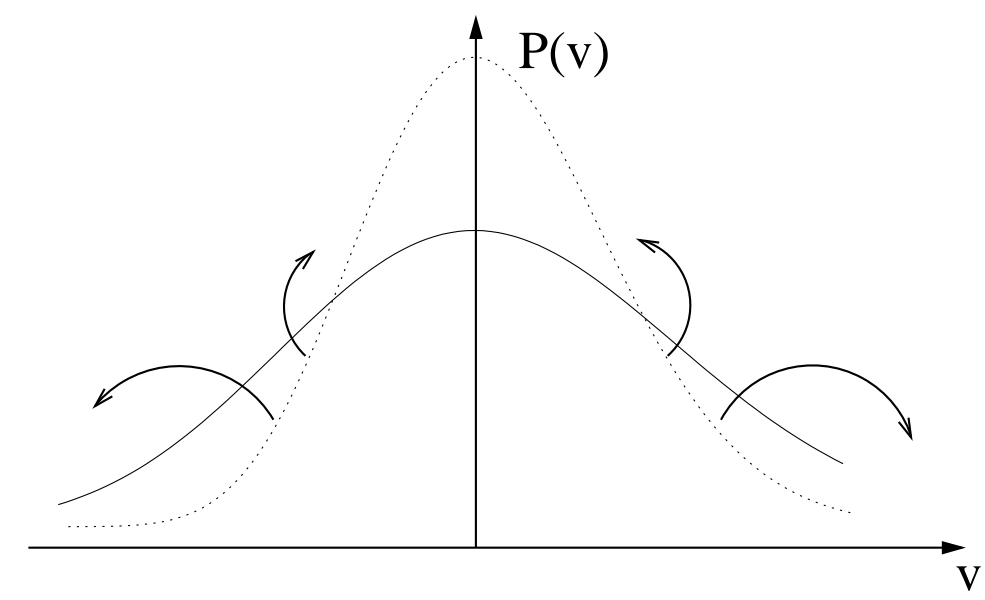

Fig. 3. Schematic evolution of the velocity distribution when clustering occurs.

ing regime has a high peak at 0 velocities and anomalously long tails. These velocity distributions have been measured by several authors and agreement exists that anomality is mainly due to having two distinct species of particles, those in the clusters and those between them.

The cooling of a dissipative gas does not lead to a steady state. One can at best formulate a scaling law in time and find self-similarity. Another way of looking at a dissipative gas is to produce a real steady state by driving the system injecting into it homogeneously some energy to balance the energy which is dissipated. The driving can be done in different ways, the most common one being componentwise additive:

$$
v(t+\Delta t)=v(t)+\eta v_{o},
$$

in which at regular intervals $\Delta t$ each particle gets a change of its velocity by a randomly chosen value. $\eta$ is typically a Gaussian random number, $v_{o}$ a reference velocity. Recently, Cafiero et al. [17] have generalised this driving to

$$
v(t+\Delta t)=v(t)+\eta|v|^{\delta} v_{o}^{1-\delta},
$$

where the case $\delta=1$ corresponds to multiplicative driving and $\delta=0$ to eq. (3).

Finally, also a driving of the rotational degree of freedom has been proposed by Cafiero et al. [18]. There, energy is injected not into the translational velocities, but into the angular velocities of the particles in an additive way

$$
\omega=\omega(t)+\eta v_{o} .
$$

The driving through the translational velocity can always lead to clustering, 


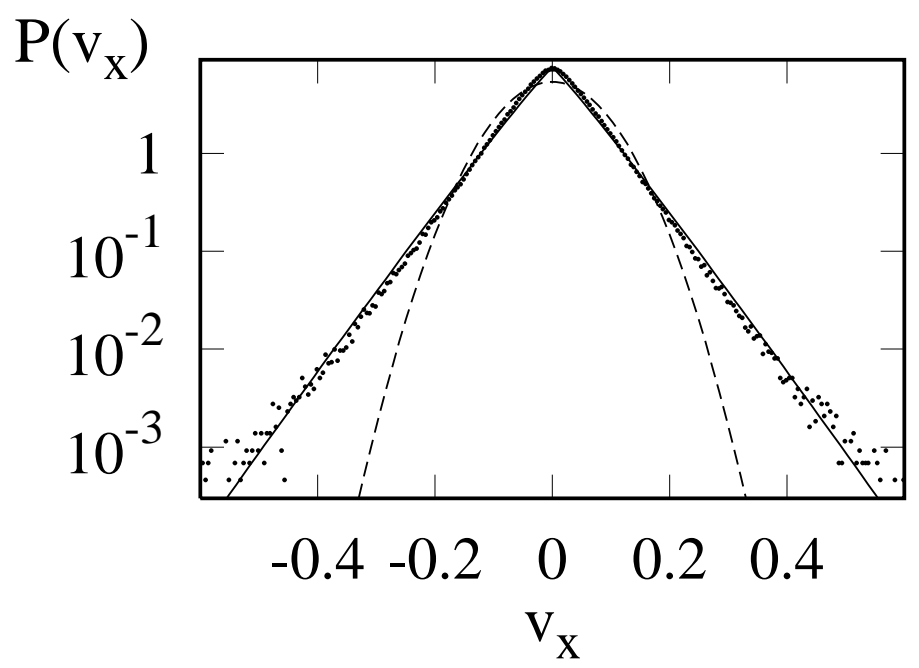

Fig. 4. Velocity distribution for $\delta=1, r=0.9$. The dashed line is a Gaussian and the full line has $f_{\mathrm{o}}=7.6, B=18.9$ and $\alpha=1.05$, see eq. (6).

where the clusters can have quite different shapes according to the values chosen for the restitution coefficient. Only in the limit of $r$ very close to unity, the system stays homogeneous and a recent mean field formulation which includes disipations formulated by Huthmann and Zippelius [19] gives an expression for the kinetic energy in the steady state as function of $r$ but only agrees with the numerical data in the region of $r$ sufficiently close to unity. The velocity distributions in Fig. 4 for the case of $\delta=1, r=0.9$ shows very strong deviations from the Gaussian behaviour - and seems to agree much better with a simple exponential decay. In fact, Cafiero et al. [17] have made a systematic analysis of the tails of the velocity distributions as function of $r$ and fitted them to a stretched exponential of the shape

$$
f_{o} \exp \left(-B\left|v_{x}\right|^{\alpha}\right)
$$

where $f_{o}, B$, and $\alpha$ are fit parameters. It was found that $\alpha=2$ in the limit $r \longrightarrow 1$ - a Gaussian distribution in the elastic limit, as expected. For small values of $r$ they observed good agreement with a mean field prediction $\alpha=$ $\frac{3-2 \delta}{2}[17,20]$. Again one would physically argue that the deviations from the Gauss distribution are mainly due to clustering. The mean field theory predicts $\alpha=3 / 2$ for additive driving and this has recently been measured by Losert et al. [5] in vibrated layers of glass beads.

The picture is quite different for the case of rotational driving. There, Cafiero et al. [18] found that the system shows no clustering instability. Fig. 5 shows an image for $r=0.1$. Thus, the system appears homogeneous at least at large scales. The mean field theory of Huthmann and Zippelius [19] works very well 


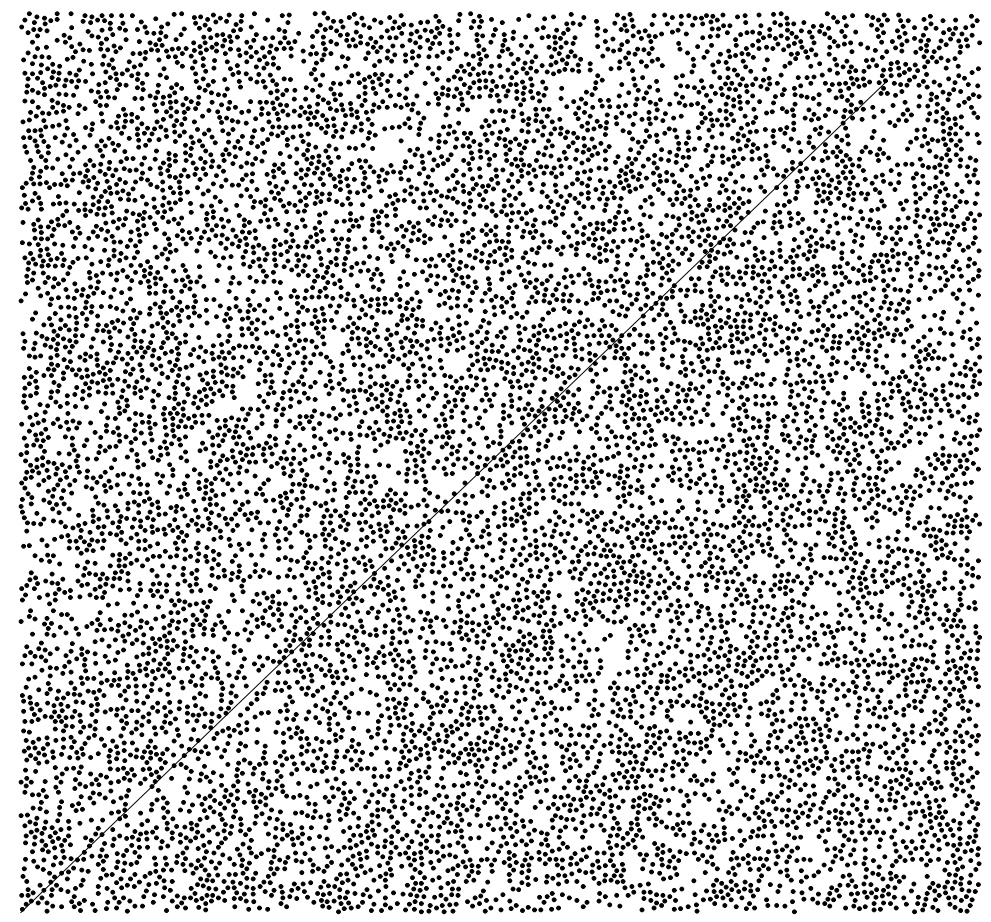

Fig. 5. Steady state for $r=0.1$ and rotational driving.

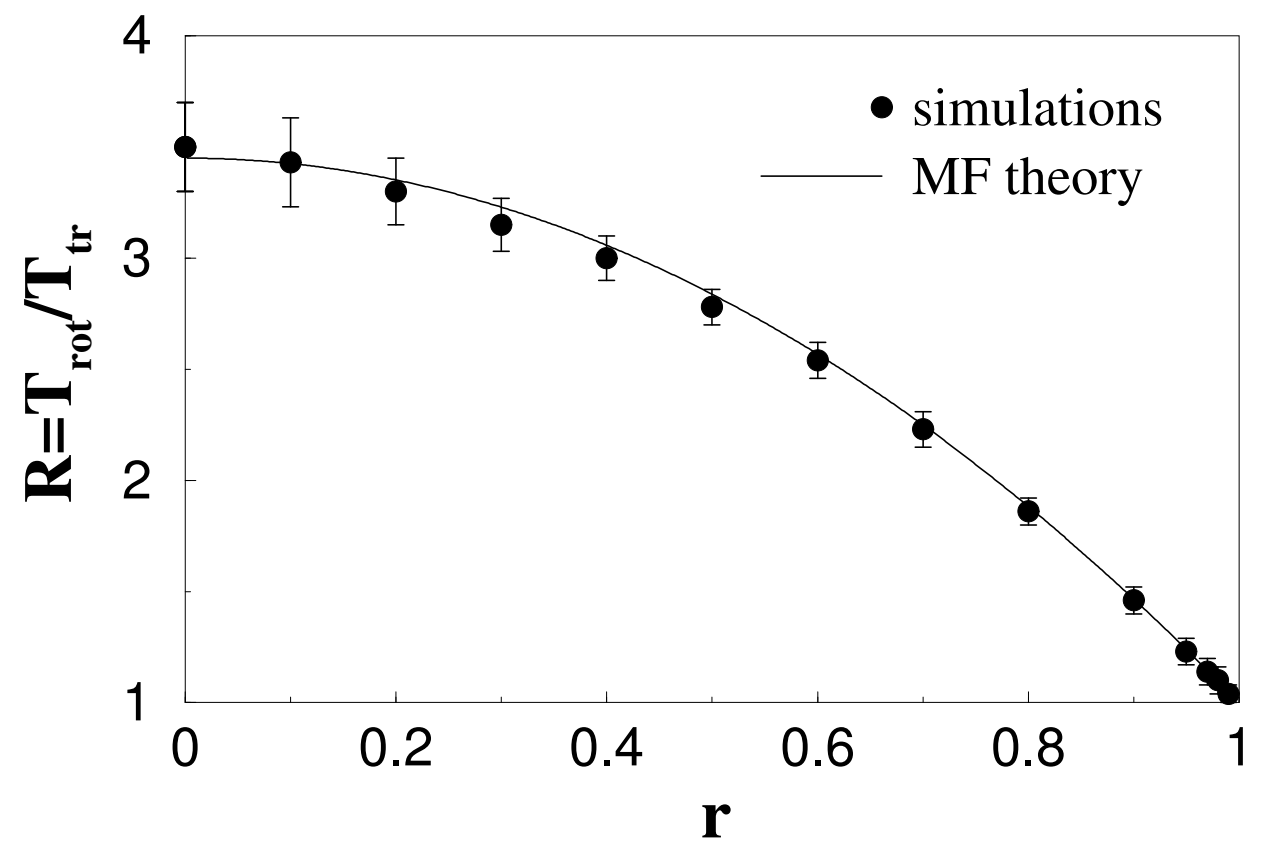

Fig. 6. Simulation (points) and mean field theory (full line) for the ratio between rotational and translational energy.

for all values of $r$, see Fig. 6 .

The distribution of angular velocities agrees quite well with a Gaussian. But the distribution of translational velocities is anomalous; the data are shown in 


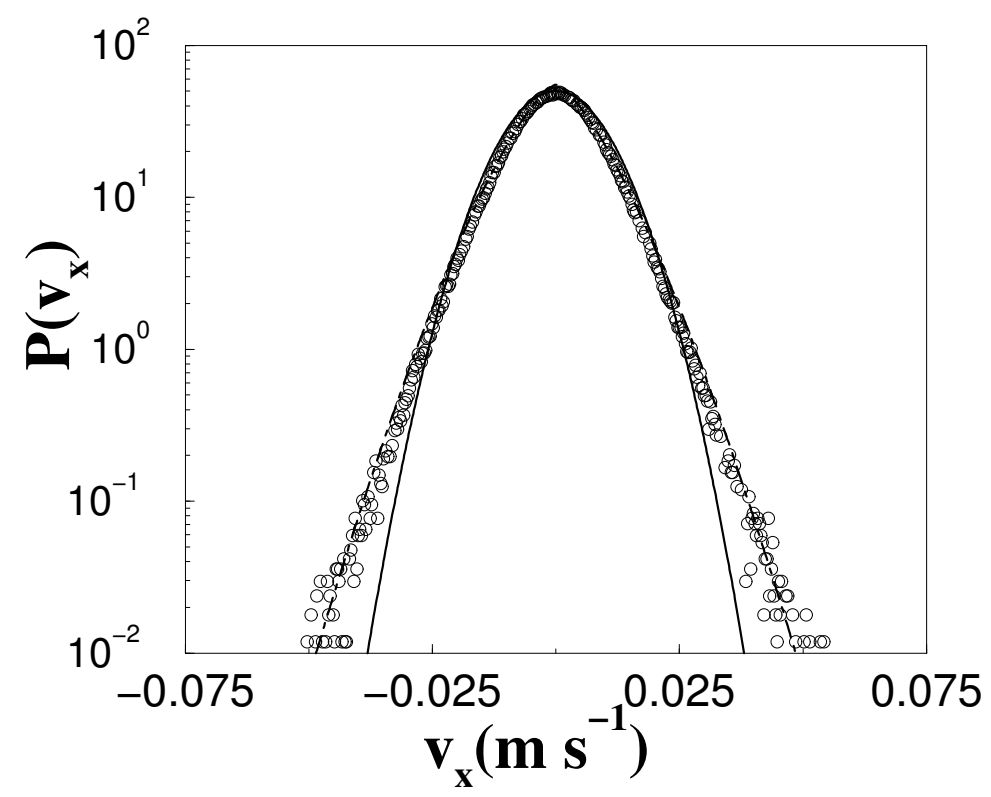

Fig. 7. Translational velocity distribution for $r=0.1$ and rotational driving. The solid line is a Gaussian and the dashed line has the form of eq. (6), with $\delta=1.41$.

Fig. 7. The line through the data points is a fit of the stretched exponential with $\alpha=1.41$. This is a very interesting result because it shows that one can find important substantial deviations from the Boltzmann behaviour without having clustering and being in a steady state with a Gaussian driving with one degree of freedom (rotation). The sole transfer from rotational to translational motion in an inelastic way is responsible for the anomaly.

Summarizing, we have described the status of a dissipative gas. The anomalous velocity distributions are not necessarily due to clustering. The dissipative gas acts as a noise transformer which transfers Gaussian into non-Gaussian distributions by going from one degree of freedom to the other without conserving energy. This observation still has to be understood.

\section{Distributions in dense granular packings}

Behringer and collaborators $[10,11,21,22]$ performed experiments in two-dimensional Couette-cells filled with about 3000 photoelastic disks and monitored in detail velocities and rotations of each individual disk during shearing in steady state. This shearing was imposed by rotating the inner wheel of the cell and keeping the outer wheel at rest. The force distributions were visualized and the density, tangential velocity and average spin were measured as functions of $R$, the distance from the inner wheel. Also the probability distributions for the tangential velocities were determined. Numerical simulations $[10,12]$ have 


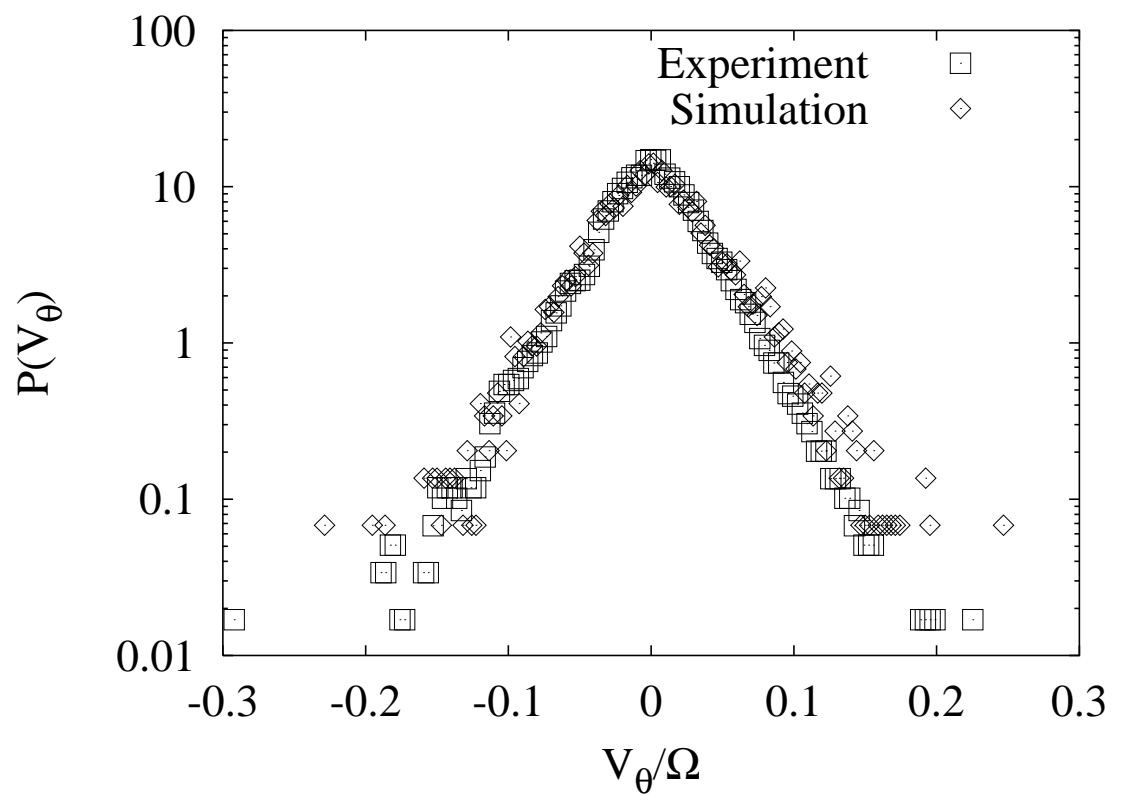

Fig. 8. Tangential velocity distribution close to the fixed boundary.

tried to reproduce as faithfully as possible the experimental conditions and obtained agreement with the experiments in all the average properties.

The result of this combination, experiments and simulations, concerning the velocity distributions is shown in Fig. 8 close to the outer ring which is at rest. There the velocities are very small since the granular packing does nearly not move. As seen in the figure, the distributions are not Gaussian, but have rather exponential tails. One also recognizes the good agreement between experiment and simulation. For the case of such dense systems, one can actually argue in favour of such an exponential distribution. Since one can suppose that the particles are equidistant, one can consider the collision rate be a fixed number without important fluctuations. Let us say that the particles collide at regular intervals and diminish each time their velocity by a factor of $r$. Supposing one is in steady state, one has the scaling behaviour $P(v)=P(r v)$ which is solved by a stretched exponential of the form $e^{-a v^{\alpha}}$. Since one can expect that the average velocity also rescales by a multiplication with $r$, one concludes $\alpha=1$ which is the searched result.

Velocity distributions measured at the inner ring are typically bimodal with one peak at zero velocity $[11,21,22]$. Such distributions were also found in MD simulations of vibrated systems [10]. 


\section{Conclusions}

In this short paper we have reviewed the empirical situation of velocity distributions in granular media. Clearly, deviation from Boltzmann statistics is observed. In the case of clustering an anomalous behaviour can be explained by the appearance of two types of particles, the slow ones inside of the cluster and the faster ones between the clusters. In the case of dense systems the lack of fluctuations in distances between particles produces exponential tails due to the multiplicative nature of dissipation. The dilute case in which a system is driven through rotations fulfils the conditions of homogeneity, molecular chaos and Gaussian distribution in the driven degrees of freedom. Nevertheless does the dissipative transfer from one degree of freedom to the other generate velocity distributions with tails which numerically agree with stretched exponentials and an exponent that varies from the mean field values at low restitution to the Gaussian behaviour in the elastic limit. This last case of anomaly has no physical explanation up to date.

It would certainly be interesting to try on the one hand to derive the long tail behaviour through driven Boltzmann-like equations by inserting not as usual Gaussian noise to get a more consistent understanding of the foundations of these distributions. It would also be interesting to see if a more generalised statistics like the one proposed by Tsallis [23] is an adequate tool to treat these systems.

\section{References}

[1] S.A. Balbus, in ASP Conf. Ser. 80, A. Ferrara, C.F. McKee, C. Heiles and P.R. Shapiro (eds.), p. 328.

[2] A. Kudrolli and J.P. Gollub, in Powders 86 Grains 97 (Balkema, Rotterdam, 1997), p.535

[3] J.S. Olafsen and J.S. Urbach, Phys.Rev.Lett. 81, 4369 (1998), condmat/9807148.

[4] J.S. Olafsen and J.S. Urbach, cond-mat/9905173 (unpublished).

[5] W. Losert and D.G.W. Cooper and J.P. Gollub, Phys. Rev. E 59, 5855 (1999) and cond-mat 9901203.

[6] X. Nie and E. Ben-Naim and S.Y. Chen, Europhys. Lett. 51, 679 (2000).

[7] F. Rouyet and N. Menon, Phys.Rev.Lett. 85, 3676 (2000)

[8] I. Goldhirsch and G. Zanetti, Phys.Rev.Lett. 70, 1619 (1993) 
[9] A. Puglisi and V. Loreto and U. Marini Bettolo Marconi and A. Vulpiani, Phys. Rev. E 59, 5582 (1999).

[10] C.T. Veje and D.W. Howell and R.P. Behringer and S. Schöllmann, in Physics of Dry Granular Media, NATO-ASI Series, H. J. Herrmann, J-P Hovi, and S. Luding (eds.), (Kluwer, Dordrecht, 1997), p. 237.

[11] D.W. Howell, Thesis Duke University 1999

[12] S. Schöllmann, Phys. Rev. E 59, 889 (1999).

[13] S. McNamara and W.R. Young, Phys.Rev. E 53, 5089 (1996).

[14] S. McNamara and S. Luding, Phys.Rev. E 58, 2247 (1998).

[15] S. Luding and H. J. Herrmann, Chaos 9, 673 (1999).

[16] P.K. Haff, J. Fluid Mech. 134, 401 (1983).

[17] R. Cafiero and S. Luding and H.J. Herrmann, Phys.Rev.Lett. 84, 6014 (2000) and preprint.

[18] R. Cafiero and S. Luding and H.J. Herrmann, preprint (2000)

[19] M. Huthmann and A. Zippelius, Phys. Rev. E 56, 6275 (1998)

[20] T.P.C. van Noije and M.H. Ernst and E. Trizac and I. Pagonabarraga, Phys. Rev. E 59, 4326 (1998); T.P.C. van Noije and M.H. Ernst, Granular Matter 1, 57 (1998).

[21] C.T. Veje and D.W. Howell and R.P. Behringer, Phys. Rev. E 59, 739 (1999).

[22] D.W. Howell and R.P. Behringer and C.T. Veje, Chaos 9, 559 (1999).

[23] C. Tsallis, J. Stat. Phys. 52, 479 (1988). 